\title{
The tritone paradox: Effects of spectral variables
}

\author{
DIANA DEUTSCH \\ University of California, San Diego, La Jolla, California
}

\begin{abstract}
A paradoxical two-tone pattern is explored, which is heard as ascending when played in one key but as descending when played in a different key. The pattern thus provides a striking counterexample to the principle of invariance under transposition. In addition, the pattern in any one key is heard as ascending by some listeners but as descending by others. This study examines the effects of spectral variables on how the pattern is perceived.
\end{abstract}

In general, when a melody is played in two different keys, the perceived relationships between the tones are unaltered. For this reason, transposition has been described as analogous to translating a shape to a different position in the visual field; the perceptual identities of the patterns are preserved under both transformations (Von Ehrenfels, 1890).

This paper concerns a two-tone pattern that provides a striking exception to the principle of invariance under transposition. It is heard as ascending in one key, but as descending in another key. As a further paradox, the pattern in any one key is heard as ascending by some listeners but as descending by others. This phenomenon is here investigated in detail, and is shown to persist under various spectral manipulations.

The research was conducted from the viewpoint that pitch consists of two components: The circular component of pitch class, or chroma, defines the position of a tone within the octave, and the rectilinear component of height defines its position on a continuum from high to low (Babbitt, 1960, 1965; Bachem, 1948; Deutsch, 1969, 1982; Forte, 1973; Meyer, 1904, 1914; Revesz, 1913; Ruckmick, 1929; Shepard, 1964, 1982; Ward \& Burns, 1982). Although traditional psychoacoustical approaches to pitch have focused almost exclusively on the latter component, the present assumption has long been considered self-evident by musicians. Thus, the Western musical scale consists of the notes, C, C $\$, \ldots, B$, formed by the division of the octave into 12 semitonal steps (Figure 1); this series of notes is repeatedly presented across octaves, and so at different pitch heights. Thus, the symbols D3, D4, and D5 represent notes that are of the same pitch class but placed in three different octaves, and the symbols D3, $F \$ 3$, and $G 3$ represent notes that are of different pitch classes but placed in the same octave. In Western tonal music, unison and octave intervals are considered har-

This work was supported by a grant from the System Development Foundation. I am indebted to F. Richard Moore for the use of the Computer Audio Research Laboratory at the UCSD Center for Music Experiment, to F. Richard Moore and Mark Dolson for software used in synthesizing the tones, and to Lee Ray and William L. Kuyper for technical assistance. Requests for reprints should be addressed to Diana Deutsch, Department of Psychology, University of California, San Diego, La Jolla, California 92093.

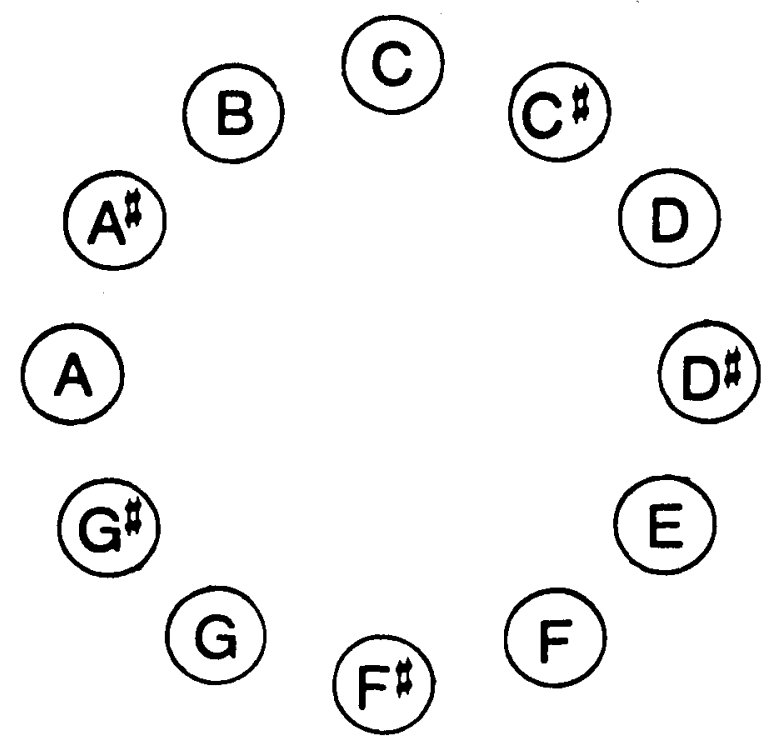

Figure 1. The pitch class circle.

monically interchangeable and chord inversions are considered equivalent to their parent chords (Piston, 1941).

Similar bidimensional representations of pitch exist in the music of other cultures. In one version of Indian musical notation, for example, a note is represented by a letter, which designates its position within the octave, together with one or more dots that designate the octave in which it is placed. Thus, the symbols $. \mathrm{m}, \mathrm{m}, \mathrm{m}, \mathrm{m}$, and $\ddot{m}$ represent notes that are of the same pitch class but placed in different octaves. Indeed, with very rare exceptions, it appears that the principle of octave equivalence holds true cross-culturally (Dowling \& Harwood, 1986; Nettl, 1956).

Experimental evidence for such a bidimensional representation may also be cited: People with absolute pitch sometimes make octave errors when assigning names to notes (Bachem, 1954; Baird, 1917; Lockhead \& Byrd, 1981; Ward \& Burns, 1982). Conditioning studies have obtained generalization of response to tones that stand in octave relation, both in human subjects (Humphreys, 1939; Demany \& Armand, 1984) and in animals (Blackwell \& Schlosberg, 1943). Interference effects in short- 
term memory for pitch exhibit octave generalization (Deutsch, 1973). Tone pairs that are related exactly by octaves are judged as closely similar in a musical context (Krumhansl, 1979).

At the theoretical level, a number of representations of pitch that accommodate both the pitch class and the height dimensions have been proposed. For example, it has been suggested that pitch should be described as a helix that completes one turn per octave, so that tones that are an octave apart are situated in close spatial proximity (M. W. Drobisch, 1846, cited in Ruckmick, 1929; Shepard, 1965, 1982). Another proposal is that the rectilinear dimension of height and the circular dimension of pitch class be represented as two separate arrays, with the pitch class array formed of projections arising from the height array (Deutsch, 1969, 1982). However, very little is known concerning how these two dimensions interact in determining the pitch of a complex tone. Rather, it has been assumed, by default, that the dimensions are orthogonal. So, for example, the statement " $G$ is higher than $D$ " is considered meaningful only if the octaves are also specified; G4 is higher than $D 4$ but lower than $D \$ 5$.

One experiment that explored the relationship between pitch class and height was performed by Shepard (1964). He employed tones that consisted of 10 octave-related sinusoidal components, the amplitudes of which differed in accordance with a fixed, bell-shaped spectral envelope. The pitch classes of the tones were varied by shifting the sinusoidal components up or down in log frequency, keeping the shape and position of the envelope constant. Because the envelope was fixed, it was predicted that the perceived heights of the tones would remain invariant in face of differences in pitch class.

Listeners were presented with ordered pairs of such tones, and they judged, for each pair, whether it formed an ascending or a descending pattern. When the tones were related by one or two steps along the pitch-class circle, judgments were overwhelmingly determined by proximity. So, for example, when $G$ was played followed by $G^{\sharp}$, the sequence was heard as ascending. Analogously, when $D$ was played followed by $C$, the sequence was heard as descending. When the tones were separated by greater distances along the pitch-class circle, the tendency to follow by proximity decreased, and when the tones were separated by exactly a half-octave, ascending and descending judgments occurred equally often.

Shepard's experiment demonstrated convincingly that for tones in which the usual cues for height attribution are missing, the perceptual system will invoke proximity in making judgments of relative height (see also Burns, 1981; Pollack, 1978; Risset, 1971; Schroeder, 1986). However, it left open the question of what happens when the cue of proximity is also removed. This situation arises, for example, when the tones within a pair are separated by exactly a half-octave (or tritone), so that the same distance along the pitch-class circle is traversed in either direction. One possibility is that the perceptual system refers to the absolute positions of the tones along the pitchclass circle. If tones in one region of the circle were tagged as higher and those in the opposite region as lower, an opportunity to resolve the ambiguity would be provided. In Shepard's experiment, the data were averaged across pitch classes, so that such a possibility was unexplored.

Deutsch (1986) performed an experiment to examine this issue, using a few musically trained subjects. Tone pairs were employed whose pitch classes were related by a half-octave, and each tone consisted of six octave-related components. Subjects judged for each pair of tones whether it formed an ascending or a descending pattern. The data were analyzed for each subject separately as a function of the pitch class of the first tone of the pair. A striking relationship between pitch class and perceived height was obtained: The judgments of most subjects depended in an orderly fashion on the pitch class of the first tone of the pair, so that tones in one region of the pitchclass circle were perceived as higher and those in the opposite region were perceived as lower. Another surprising finding was that the direction of the relationship between pitch class and perceived height varied considerably among subjects.

Deutsch, Kuyper, and Fisher (1987) investigated this phenomenon in a large group of subjects who were unselected with regard to musical training, the only selection criteria being that the subjects have normal hearing and be able to judge reliably whether pairs of sine-wave tones formed ascending or descending patterns. The judgments of the large majority of these subjects were found to reveal an orderly relationship between pitch class and perceived height, showing that this phenomenon is not confined to a specialized group of listeners.

The experiments of Deutsch (1986) and Deutsch et al. (1987) employed envelopes that were placed at several different positions along the spectrum and spaced at halfoctave intervals. The results obtained under these different spectral conditions were then averaged. This procedure provided an internal control for explanations of the phenomenon in terms of the relative amplitudes of the sinusoidal components of the tones. However, it left unexplored possible superimposed effects of variations in the position of the spectral envelope. Such variations could, in principle, produce effects in two ways: first, through resultant differences in the overall heights of the tones, and second, through resultant differences in the relative amplitudes of their components.

The present study was undertaken to examine both these possibilities. In Experiment 1, eight spectral envelopes were employed, whose peaks were spaced at $1 / 4$-octave intervals, so encompassing a 2-octave range. The employment of these envelopes enabled a partitioning of the effects of the relative amplitudes of the components of the tones from their overall heights. In Experiment 2, four further envelopes were employed, again with peaks spaced 
at $1 / 4$-octave intervals, encompassing a further octave's range. Thus, the effects of varying the envelope position were explored altogether over 3 octaves.

\section{EXPERIMENT 1}

\section{Method}

Stimulus patterns. Each tone consisted of 6 sinusoids that were related by octaves, and their amplitudes were determined by a fixed, bell-shaped spectral envelope. The general form of the equation describing the envelope is

$$
A(f)=0.5-.05 \cos \left[\frac{2 \pi}{\gamma} \log _{\beta}\left(\frac{f}{f_{\min }}\right)\right] \quad f_{\min } \leq f \leq \beta^{\gamma} f_{\min }
$$

where $A(f)$ is the relative amplitude of a sinusoid at frequency of $f \mathrm{~Hz}, \beta$ is the frequency ratio formed by adjacent sinusoids (so that for octave spacing, $\beta=2$ ), $\gamma$ is the number of $\beta$ cycles spanned, and $f_{\min }$ is the minimum frequency for which the amplitude is nonzero. Thus, the maximum frequency for which the amplitude is nonzero is $\gamma \beta$ cycles above $f_{\min }$. The values $\beta=2$ and $\gamma=6$ were used throughout, so that the spectral envelope always spanned exactly 6 octaves from $f_{\min }$ to $64 f_{\min }$.

This envelope was placed at eight different positions along the spectrum, with peaks spaced at $1 / 4$-octave intervals, so encompassing a 2-octave range. Figure 2 displays the four positions of the envelope within the higher octave, with peaks at $C 5\left(523 \mathrm{~Hz}, f_{\min }\right.$ $=65.4 \mathrm{~Hz}), \mathrm{D} \sharp 5\left(622 \mathrm{~Hz}, f_{\min }=77.7 \mathrm{~Hz}\right), \mathrm{F} \sharp 5\left(740 \mathrm{~Hz}, f_{\min }\right.$ $=92.4 \mathrm{~Hz})$, and $\mathrm{A} 5\left(880 \mathrm{~Hz}, f_{\min }=110 \mathrm{~Hz}\right)$. Figure 3 displays the four positions of the envelope within the lower octave, with peaks at $\mathrm{C} 4\left(262 \mathrm{~Hz}, f_{\min }=32.7 \mathrm{~Hz}\right), \mathrm{D} \$ 4\left(311 \mathrm{~Hz}, f_{\min }=\right.$
$38.8 \mathrm{~Hz}), \mathrm{F} \# 4\left(370 \mathrm{~Hz}, f_{\min }=46.2 \mathrm{~Hz}\right)$, and $\mathrm{A} 4\left(440 \mathrm{~Hz}, f_{\min }\right.$ $=55.0 \mathrm{~Hz}$ ). It can be seen that the relative amplitudes of the sinusoidal components of tones generated under the C5 envelope were identical to those generated under the $\mathrm{C} 4$ envelope. ${ }^{1}$ The same held true for the $D \$ 5$ and $D \$ 4$ envelopes, for the $F \$ 5$ and $F \$ 4$ envelopes, and for the A5 and A4 envelopes. This enabled the partitioning of the effects of overall heights of the tones from those of the relative amplitudes of their sinusoidal components.

It should also be noted that for tones at any pitch class, the relative amplitudes of the sinusoidal components when generated under a $\mathrm{C}$ envelope were identical to those at the pitch class a halfoctave removed when generated under an F envelope. Similarly, for tones at any pitch class, the relative amplitudes of the components when generated under a $\mathrm{D}$ \# envelope were identical to those at the pitch class a half-octave removed when generated under an $A$ envelope. Thus, when results obtained under $C, D \#, F H$, and A envelopes were averaged, these relationships enabled a balancing out of the effects of the relative amplitudes of the sinusoidal components of the tones.

Twelve ordered pairs of tones were generated under each of the eight spectral envelopes, corresponding to the pitch-class pairings $C-F \$, C \$-G, D-G \$, D \#-A, E-A \$, F-B, F \$-C, G-C \#, G \$-D, A-D \#$, $A-E$, and $B-F$. Ninety-six ordered tone pairs were thus produced altogether. These were presented in blocks of 12 , each block consisting of tones generated under one of the spectral envelopes and containing one example of each of the 12 pitch-class pairings. Within blocks, the tone pairs were presented in any of four orders. These were random, with the restriction that no two consecutive pairs be composed of the same pitch classes. Thus, 32 blocks were created altogether. These were presented across two sessions, each session consisting of 16 blocks. The entire procedure was repeated three times, so that each subject participated in six sessions.



Figure 2. Representation of the spectral composition of the tones comprising a D-G pattern in Experiment 1 , generated under the four envelopes centered in the higher octave. Dashed lines indicate tones of pitch class $D$, and solid lines indicate tones of pitch class $G \not ̈$. The two sets of spectra are superimposed in the illustration, although the tones were presented in succession. 


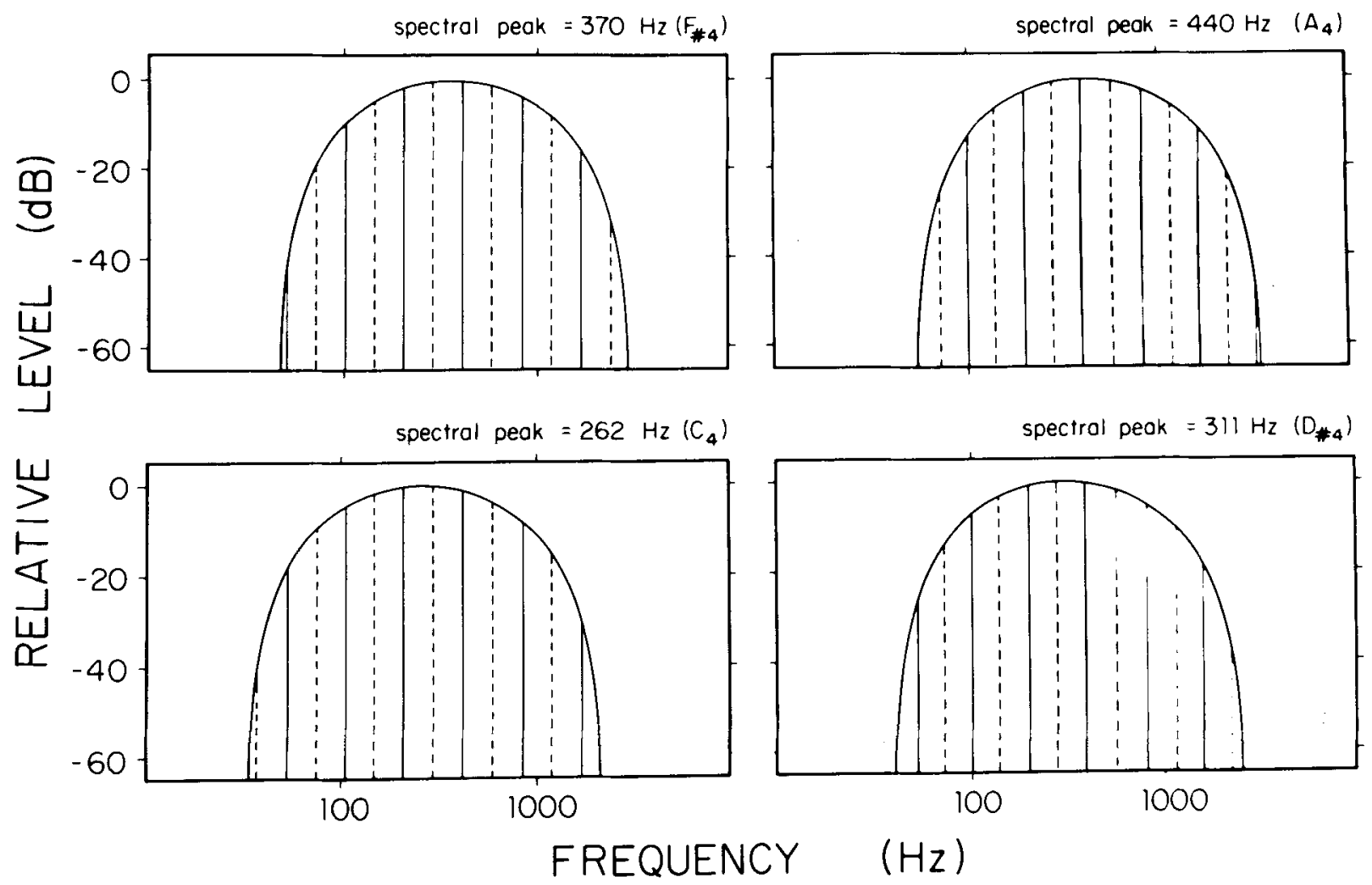

Figure 3. Representation of the spectral composition of the tones comprising a D-G pattern in Experiment 1, generated under the four envelopes centered in the lower octave. Dashed lines indicate tones of pitch class $D$, and solid lines indicate tones of pitch class $G$. The two sets of spectra are superimposed in the illustration, although the tones were presented in succession.

Procedure. The subjects were tested in soundproof booths. On each trial, an ordered pair of tones was presented and the subject judged whether it formed an ascending or a descending pattern. All tones were $500 \mathrm{msec}$ in duration, and there were no gaps between tones within a pair. Trials within a block were separated by 5-sec pauses, and blocks were separated by 1-min pauses. A break of 5 min intervened between the 8th and 9th blocks. A few warmup trials were given at the beginning of each session.

Equipment. The tones were generated on a VAX 11/780 computer, interfaced with a DSC-200 Audio Data Conversion System and using the cmusic sound synthesis software (Moore, 1982). They were recorded and played back on a Sony PCM-F1 digital audio processor, the output of which was passed through a Crown amplifier and presented to listeners binaurally through headphones (Grason-Stadler TDH-49) at a level of approximately $72 \mathrm{~dB}$ SPL.

Subjects. Four listeners with normal hearing served as subjects in the experiment, and they were paid for their services. The subjects were selected for showing a clear influence of pitch class on perceived height in a brief preliminary experiment employing tones generated under a small subset of the envelopes employed here. None of the subjects had absolute pitch in the sense of being able to attach verbal labels to notes presented in isolation. One subject (T.K.) was a graduate student in the Music Department at the University of California, San Diego. The remaining subjects were undergraduates; two (S.F. and M.D.) with musical training, and one (P.S.) without.

\section{Results}

The percentage of judgments that a tone pair formed a descending pattern was plotted as a function of the pitch class of the first tone of the pair. Figures 4 and 5 display the results for each subject separately. Those in Figure 4 were averaged over the four envelopes centered in the higher octave (C5, D $\$ 5, F \$ 5$, and A5); those in Figure 5 were averaged over envelopes centered in the lower octave (C4, D 4 F F $\$$, and $A 4)$. A number of characteristics are readily apparent from these averaged data. First, each subject showed a highly systematic effect of position of the first tone along the pitch-class circle, so that tones in one region of the circle were heard as higher and those in the opposite region were heard as lower. Second, for each subject, the direction of the relationship between pitch class and perceived height was remarkably similar across the 2 octaves. Third, the direction of the relationship between pitch class and perceived height varied considerably across subjects.

Figures 6-9 display the results for each subject separately, as a function of the pitch class of the peak of the spectral envelope, and so of the relative amplitudes of the sinusoidal components. It can be seen that, for the most part, the profiles relating pitch class to perceived height were very similar in the face of this manipulation.

For each subject, a four-way ANOVA was performed, with pitch class of the first tone (pitch class), octave of the peak of the spectral envelope (overall height), and pitch class of the peak of the spectral envelope (relative amplitude) as fixed factors and replications as a random 

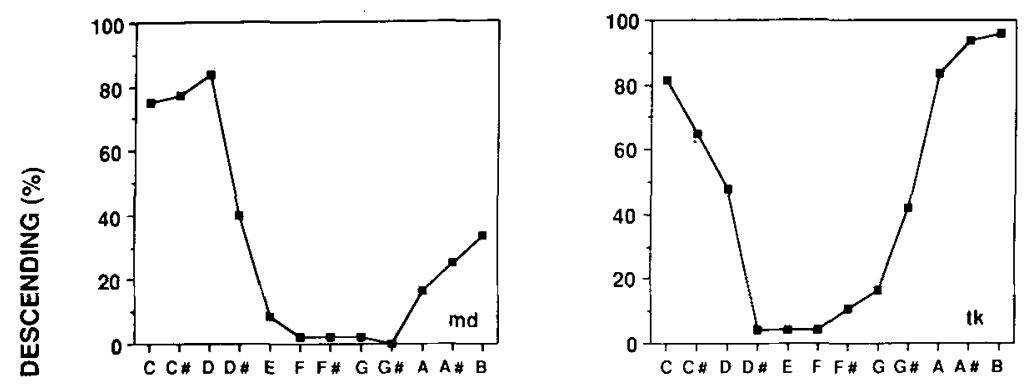

离
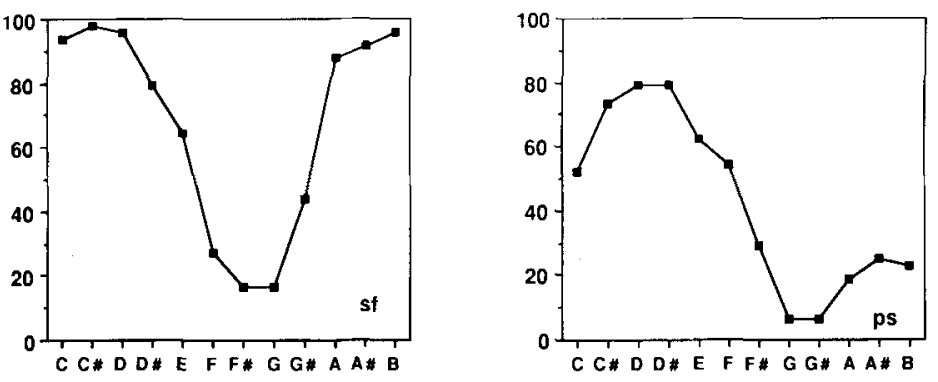

PITCH CLASS OF FIRST TONE

Figure 4. Percentages of judgments that a tone pair formed a descending pattern, plotted as a function of the pitch class of the first tone of the pair. The results are plotted for each subject separately, averaged over the four envelopes centered in the higher octave, that is, with spectral peaks at C5, D 5, F 5 , and A5.


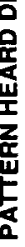
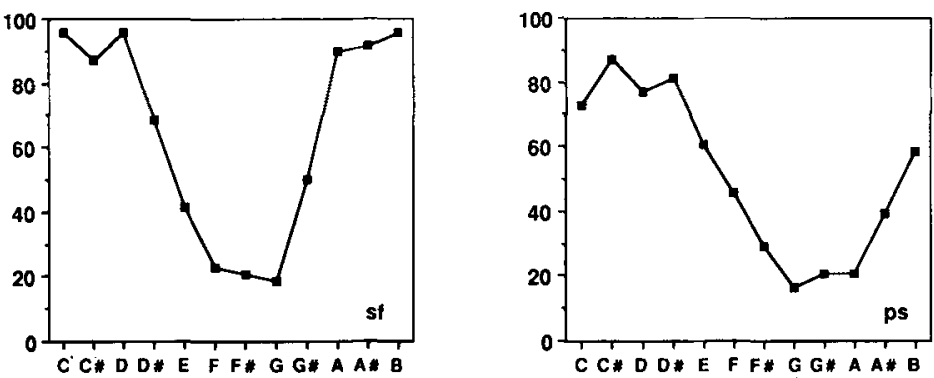

PITCH CLASS OF FIRST TONE

Figure 5. Percentages of judgments that a tone pair formed a descending pattern, plotted as a function of the pitch class of the first tone of the pair. The results are plotted for each subject separately, averaged over the four envelopes centered in the lower octave, that is, with spectral peaks at $\mathrm{C} 4, \mathrm{D} \$ 4, \mathrm{~F} \$ 4$, and $\mathrm{A4}$. 

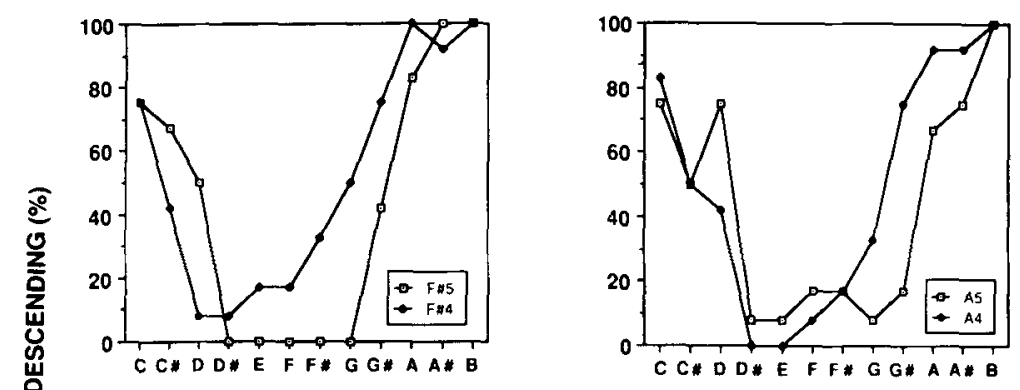

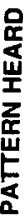
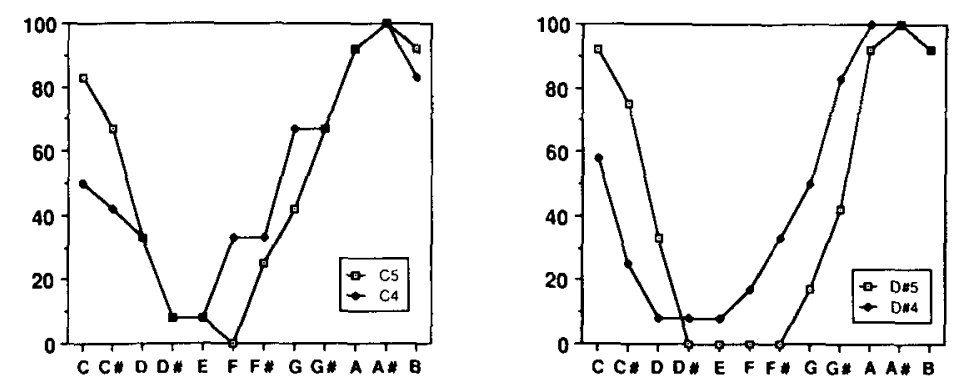

PITCH CLASS OF FIRST TONE

Figure 6. Percentages of judgments that a tone pair formed a descending pattern, plotted as a function of the pitch class of the first tone of the pair. The results are plotted separately by the pitch class of the peak of the spectral envelope, in both the higher and the lower octaves. The results are from Subject T.K.
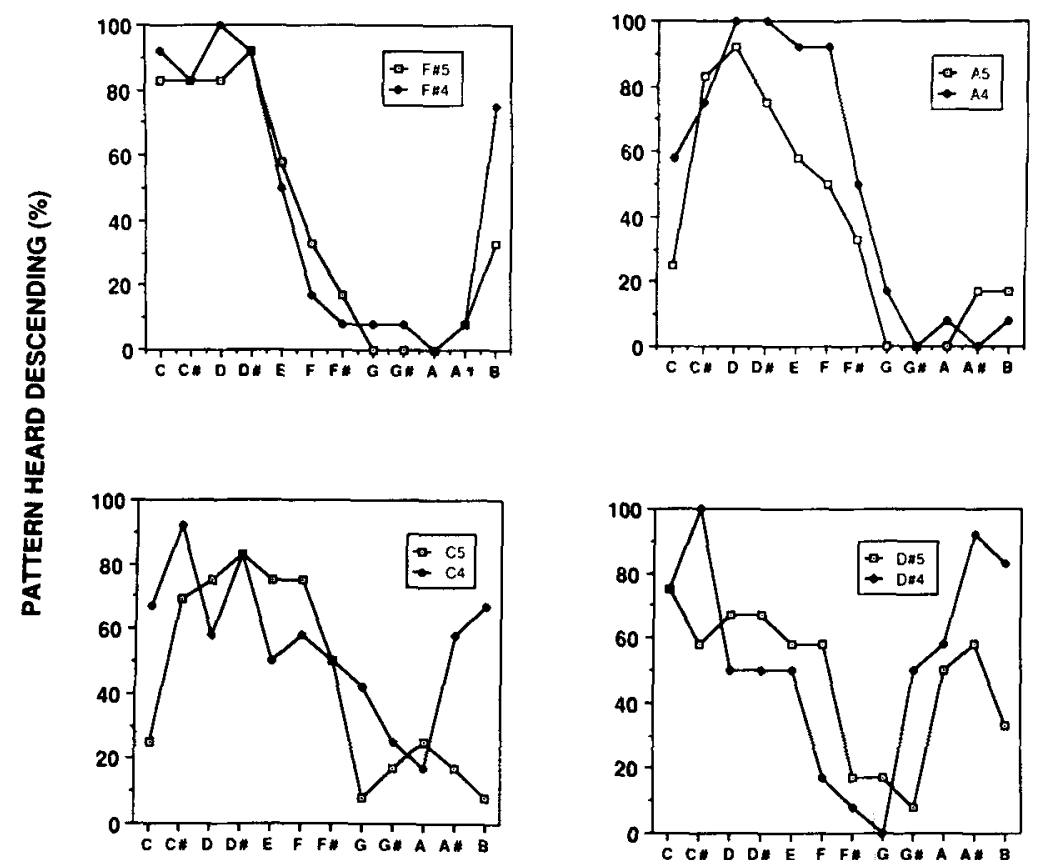

PITCH CLASS OF FIRST TONE

Figure 7. Percentages of judgments that a tone pair formed a descending pattern, plotted as a function of the pitch class of the first tone of the pair. The results are plotted separately by the pitch class of the peak of the spectral envelope, in both the higher and the lower octaves. The results are from Subject P.S. 



PITCH CLASS OF FIAST TONE

Figure 8. Percentages of judgments that a tone pair formed a descending pattern, plotted as a function of the pitch class of the first tone of the pair. The results are plotted separately by the pitch class of the peak of the spectral envelope, in both the higher and the lower octaves. The results are from Subject S.F.



Figure 9. Percentages of judgments that a tone pair formed a descending pattern, plotted as a function of the pitch class of the first tone of the pair. The results are plotted separately by the pitch class of the peak of the spectral envelope, in both the higher and the lower octaves. The results are from Subject M.D. 
factor (2). For Subject T.K., the main effect of pitch class was highly significant $[F(11,22)=72.61, p<.0001]$; no other main effects were significant. The interaction between pitch class and overall height was highly significant $[F(11,22)=7.79, p<.0001]$; no other interactions were significant. For Subject P.S., the main effect of pitch class was highly significant $[F(11,22)=65.61, p<$ $.0001]$; no other main effects were significant. The interaction between pitch class and relative amplitude was highly significant $[F(33,66)=4.08, p<.0001]$; no other interactions were significant. For Subject S.F., the main effect of pitch class was highly significant $[F(11,22)=$ $45.91, p<.0001$ ]; no other main effects were significant. The interaction between pitch class and relative amplitude was highly significant $[F(33,66)=5.31, p<$ $.0001]$; no other interactions were significant. For Subject M.D., the main effect of pitch class was highly significant $[F(11,22)=188.93, p<.0001]$; no other main effects were significant. The interaction between pitch class and overall height was highly significant $[F(11,22)$ $=7.09, p<.0001]$, as was the interaction between pitch class and relative amplitude $[F(33,66)=4.79, p<$ $.0001]$. No other interactions were significant.

The results show, therefore, that both the overall height of the spectral envelope and the relative amplitudes of the sinusoidal components can influence the effect of pitch class on perceived height; however, neither of these fac- tors necessarily does so. Thus, Subject T.K. showed a significant influence of overall height but not of relative amplitude, whereas Subjects P.S. and S.F. showed a significant influence of relative amplitude but not of overall height. Furthermore, as shown in Figures 6-9, the influence of these spectral factors was always small in absolute terms.

\section{EXPERIMENT 2}

In Experiment 1, the position of a tone along the pitchclass circle was found to exert a considerable influence on its perceived height when spectral envelopes were employed whose positions varied over 2 octaves. Experiment 2 was undertaken to examine further the generality of this phenomenon, by employing tones generated under envelopes whose positions varied over a further octave.

\section{Method}

The shape of the spectral envelope employed was identical to that used in Experiment 1. As shown in Figure 10, the envelope was placed at four different positions along the spectrum. These were spaced at 1/4-octave intervals, so that the peaks stood at C3 $(131 \mathrm{~Hz}$, $\left.f_{\min }=16.3 \mathrm{~Hz}\right), \mathrm{D} \$ 3\left(156 \mathrm{~Hz}, f_{\min }=19.4 \mathrm{~Hz}\right), \mathrm{F} \$ 3(185 \mathrm{~Hz}$, $\left.f_{\min }=23.1 \mathrm{~Hz}\right)$, and $\mathrm{A} 3\left(220 \mathrm{~Hz}, f_{\min }=27.5 \mathrm{~Hz}\right)$. Thus, the relative amplitudes of the components of the tones were identical to those in Experiment 1.



Figure 10. Representation of the spectral composition of the tones comprising a D-G pattern in Experiment 2, generated under the four envelopes. Dashed lines indicate tones of pitch class $D$, and solid lines indicate tones of pitch class $G_{k}^{\sharp}$. The two sets of spectra are superimposed in the illustration, although the tones were presented in succession. 
Twelve ordered pairs of tones were generated under each of the four envelopes, corresponding to the pitch-class pairings $C-F$. C G, ... B-F, as in Experiment 1. This produced 48 ordered tone pairs in all. They were presented in blocks of 12 , each block consisting of tone pairs generated under one of the envelopes and containing one example of each of the 12 pitch-class pairings. Within blocks, the tone pairs were presented in any of four orders. These were random, with the restriction that no consecutive pairs were composed of the same pitch classes. Sixteen blocks were thus created altogether, and these were presented in a single session. The entire procedure was repeated three times, so that each subject participated in three sessions. The method was otherwise identical to that in Experiment 1, and the same subjects were employed

\section{Results}

Figure 11 displays the results for each subject separately, averaged over the four envelopes (centered at $C 3, D \$ 3, F \$ 3$, and $A 3$ ). It can be seen that, in general, the profiles relating perceived height to the positions of the tones along the pitch-class circle were very similar to those in Experiment 1. Figures 12-15 display the results for tones generated under each of the envelopes separately. It can be seen that, on the whole, the profiles were quite similar, although individual differences in consistency of the effect were also apparent.

For each subject, a three-way ANOVA was performed, with pitch class of the first tone of the pair (pitch class) and position of the spectral envelope (envelope position) as fixed factors and replications as a random factor. For
Subject T.K., the main effect of pitch class was highly significant $[F(11,22)=44.82, p<.0001]$; the main effect of envelope position was nonsignificant. The interaction between pitch class and envelope position was highly significant $[F(33,66)=7.09, p<.0001]$. For Subject P.S., the main effect of pitch class was highly significant $[F(11,22)=29.90, p<.0001]$; the main effect of envelope position was nonsignificant. The interaction between pitch class and envelope position was highly significant $[F(33,66)=7.23, p<.0001]$. For Subject S.F., the main effect of pitch class was highly significant $[F(11,22)=13.90, p<.0001] ;$ the main effect of envelope position was nonsignificant. The interaction between pitch class and envelope position was nonsignificant. For Subject M.D., the main effect of pitch class was highly significant $[F(11,22)=20.04, p<.0001]$; the main effect of spectral envelope was nonsignificant. The interaction between pitch class and envelope position was nonsignificant.

The findings of Experiment 2 extend those of Experiment 1 to show, therefore, that the influence of pitch class on perceived height persists over a 3-octave range, and that individual profiles relating pitch class to perceived height persist for the most part throughout this range. The findings show further that there may be a superimposed effect of envelope position in the lower range also; however, this is not necessarily the case. For Subjects T.K. and P.S. there was a highly significant inter-
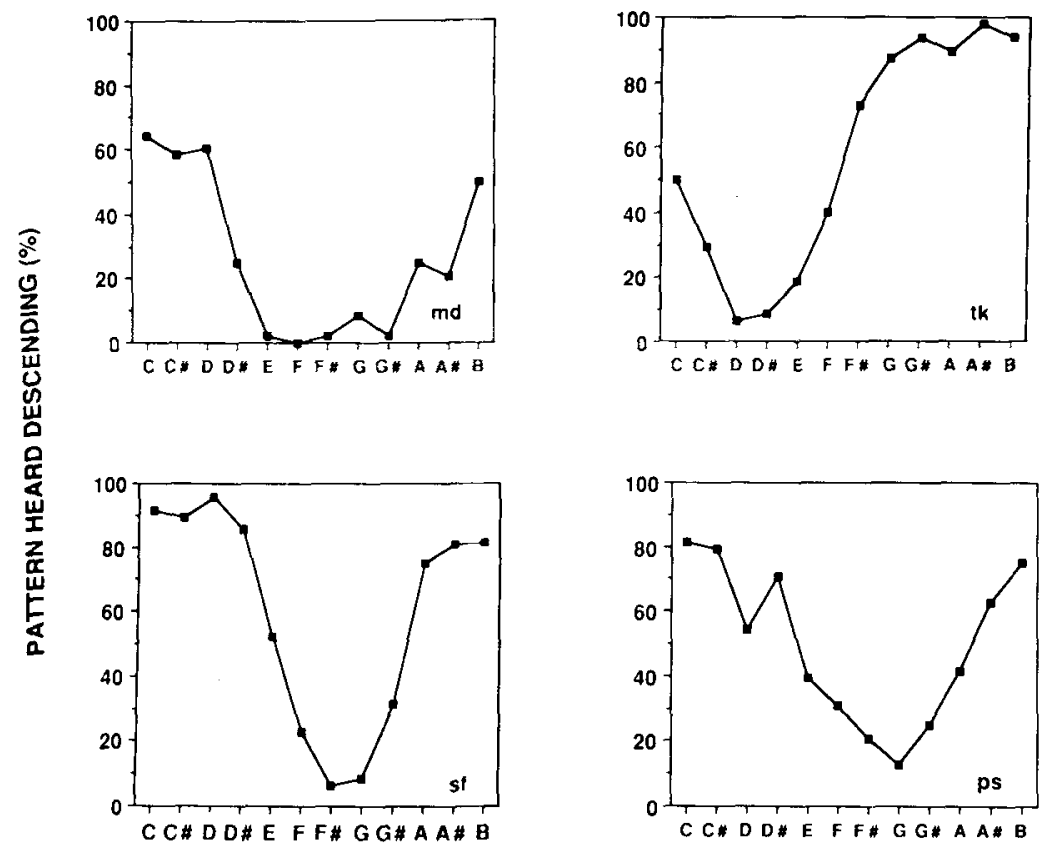

PITCH CLASS OF FIRST TONE

Figure 11. Percentages of judgments that a tone pair formed a descending pattern, plotted as a function of the pitch class of the first tone of the pair. The results are plotted for each subject separately, averaged over envelopes with spectral peaks at $C 3, D \sharp 3, F \$ 3$, and $\mathbf{A 3}$. 



PITCH CLASS OF FIRST TONE

Figure 12. Percentages of judgments that a tone pair formed a descending pattern, plotted as a function of the pitch class of the first tone of the pair. The results are plotted for each envelope position separately. The results are from Subject T.K.

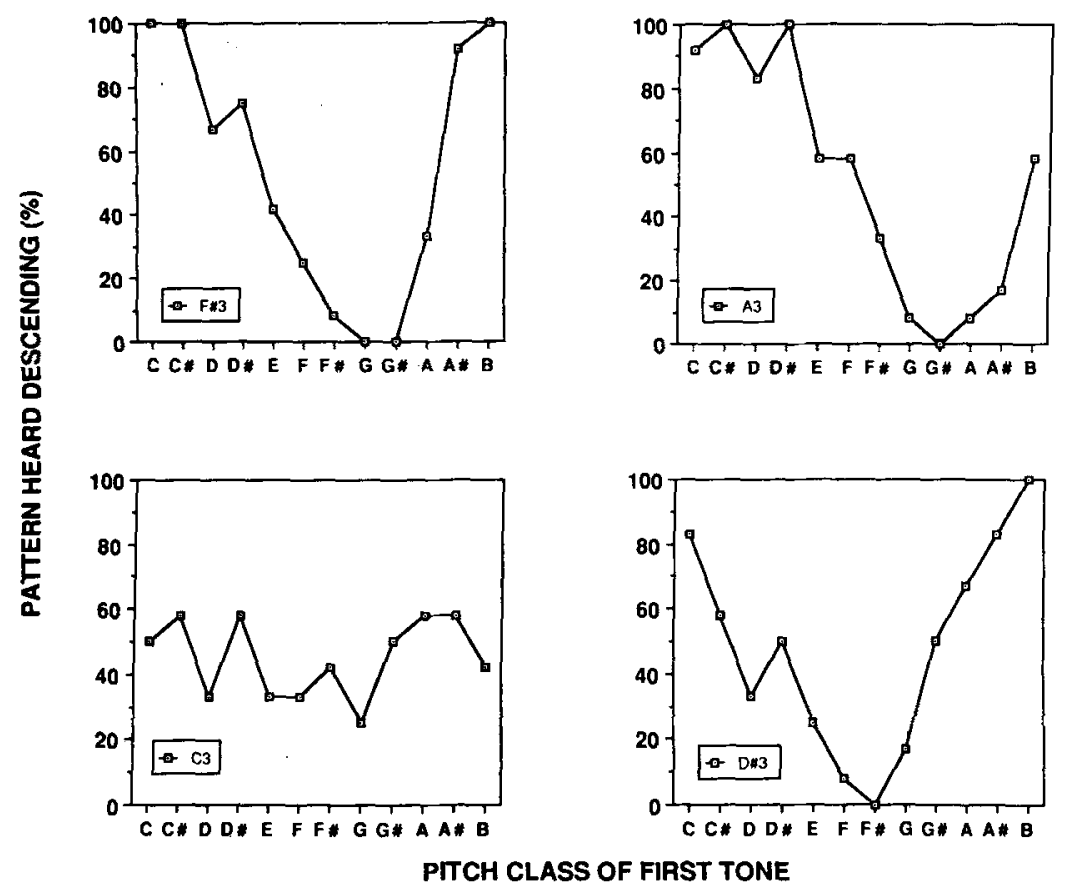

Figure 13. Percentages of judgments that a tone pair formed a descending pattern, plotted as a function of the pitch class of the first tone of the pair. The results are plotted for each envelope position separately. The results are from Subject P.S. 



㭊
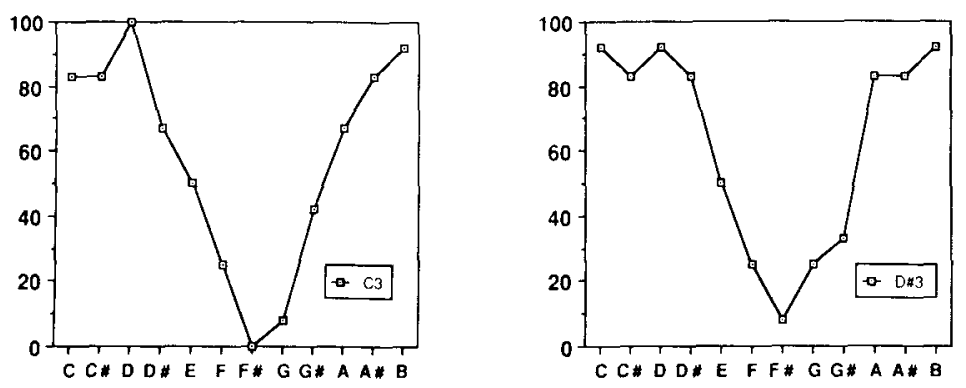

PITCH CLASS OF FIRST TONE

Figure 14. Percentages of judgments that a tone pair formed a descending pattern, plotted as a function of the pitch class of the first tone of the pair. The results are plotted for each envelope position separately. The results are from Subject S.F.

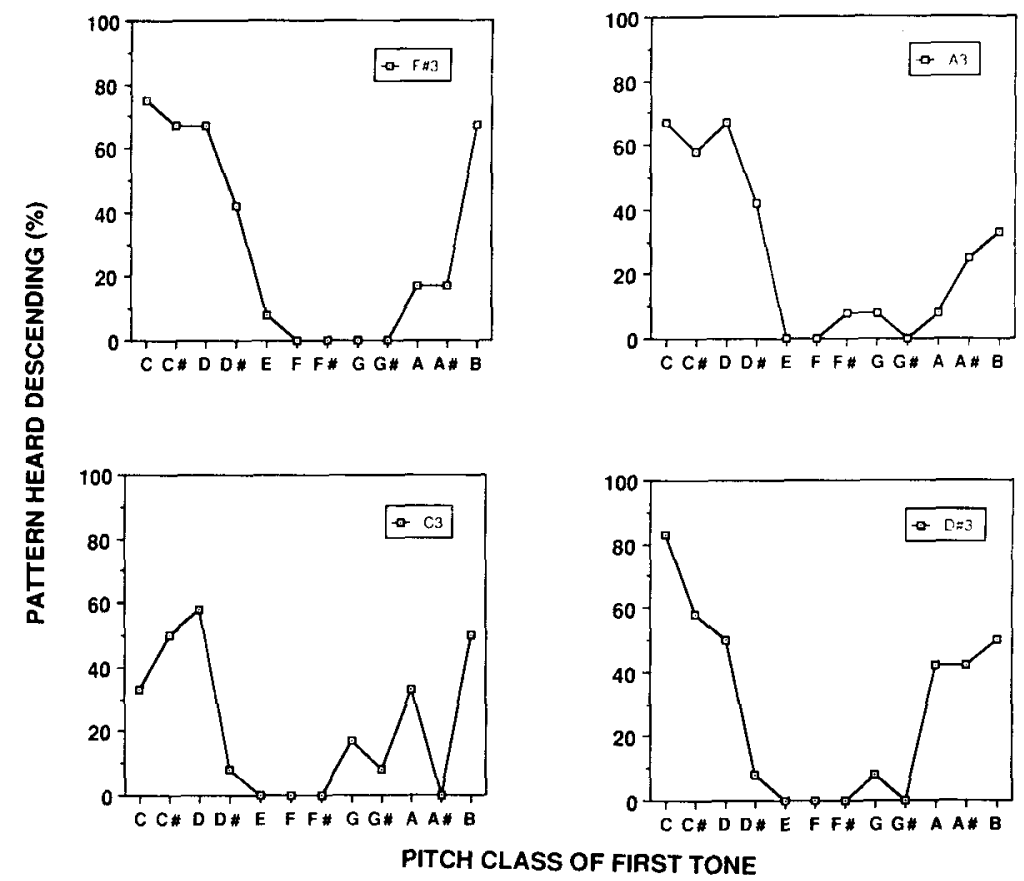

Figure 15. Percentages of judgments that a tone pair formed a descending pattern, plotted as a function of the pitch class of the first tone of the pair. The results are plotted for each envelope position separately. The results are from Subject M.D. 
action between the effects of pitch class and of envelope position; for Subjects S.F. and M.D. no significant interaction was obtained.

\section{GENERAL DISCUSSION}

The results of the present study demonstrate that, for octave-related complexes, the perceived height of a tone can be strongly influenced by its position along the pitchclass circle. This influence can result in violations of the principle of equivalence under transposition, as exemplified by the tritone paradox explored here. The present findings corroborate those obtained previously by Deutsch (1986) and Deutsch et al. (1987). In addition, they show that the form of relationship between pitch class and perceived height can be surprisingly robust in face of substantial differences in both the relative amplitudes of the sinusoidal components of the tones and their overall heights.

The present findings demonstrate further that the direction of the relationship between pitch class and perceived height can vary substantially across individual subjects. Again, these findings corroborate those obtained earlier by Deutsch (1986) and Deutsch et al. (1987). A further source of individual variation obtained here concerns the influence of spectral parameters. Some subjects were clearly influenced by the overall heights of the tones, but not necessarily by the relative amplitudes of the components; others were strongly influenced by the latter factor but not by the former. In addition, the subjects were found to differ considerably in their overall susceptibility to spectral influences.

A related influence of pitch class on perceived height was recently reported by Deutsch, Moore, and Dolson (1984, 1986). Two-part patterns consisting of octaverelated complexes were here employed, and such patterns were found to be perceived quite differently, depending on which of two keys they were in. The relative heights of the individual tones were preserved under transposition, with the result that there was a perceived interchange of voices. As with the present phenomenon, this effect was found to be robust when the position of the spectral envelope was varied over a considerable range. Furthermore, striking differences between subjects were also obtained in the direction of the relationship between pitch class and perceived height.

One surprising conclusion to emerge from this series of experiments is that, since judgments of height were determined by the positions of the tones along the pitch-class circle, listeners were, in effect, employing absolute pitch in making their judgments. Given this evident ability to utilize absolute pitch indirectly, it is most puzzling that people do not, in general, possess absolute pitch, as defined by the ability to attach verbal labels to notes heard in isolation. A similar point has been raised by Terhardt and Ward (1982) and Terhardt and Seewann (1983).
These authors found that musicians were able to judge accurately whether or not passages were played in the correct key, even though they did not possess absolute pitch in the conventional sense. We may speculate that most people have a pitch equivalent of the syndrome of color anomia, in which performance on nonverbal color tasks may be normal, but there is an inability to name colors presented in isolation (Geschwind \& Fusillo, 1966).

The mechanisms responsible for these paradoxical findings are at present unknown. It remains to be determined whether the relationship between pitch class and perceived height is confined to octave-related complexes, or whether it may also be present under different conditions in which tones are poorly defined in terms of height. Similarly, it is an open question as to whether this phenomenon might appear to some extent in the perception of music performed by natural instruments (perhaps, for example, in certain multivoiced orchestral contexts). Such issues aside, the intriguing possibility has now arisen, for computersynthesized tones at least, of producing music that not only sounds quite different under transposition, but also sounds radically different from one member of an audience to another.

\section{REFERENCES}

BABbitT, M. (1960). Twelve-tone invariants as compositional determinants. Muscial Quarterly, 46, 246-259.

BabitT, M. (1965). The structure and function of music theory. $\mathrm{Col}$ lege Music Symposium, 5, 10-21.

BACHEM, A. (1948). Note on Neu's review of the literature on absolute pitch. Psychological Bulletin, 45, 161-162.

BACHEM, A. (1954). Time factors in relative and absolute pitch determination. Journal of the Acoustical Society of America, 26, 751-753.

BAIRD, J. W. (1917). Memory for absolute pitch: Studies in psychology. In Titchener Commemorative Volume. Worcester.

BLACKWELL, H. R., \& SCHLOSBERG, H. (1943). Octave generalization, pitch discrimination and loudness thresholds in the white rat. Journal of Experimental Psychology, 33, 407-419.

BURNs, E. (1981). Circularity in relative pitch judgments for inharmonic tones: The Shepard demonstration revisited, again. Perception \& Psychophysics, 30, 467-472.

Demany, L., \& ARMAND, F. (1984). The perceptual reality of tone chroma in early infancy. Journal of the Acoustical Society of America, 76, 57-66.

Deutsch, D. (1969). Music recognition. Psychological Review, 76, 300-307.

DEUTSCH, D. (1973). Octave generalization of specific interference effects in memory for tonal pitch. Perception \& Psychophysics, 13, 271-275.

DEuTsCH, D. (1982). The processing of pitch combinations. In D. Deutsch (Ed.), The psychology of music. New York: Academic Press.

Deutsch, D. (1986). A musical paradox. Music Perception, 3, 275-280.

DEUTSCH, D., KUYPER, W. L., \& FISHER, Y. (1987). A new characteristic of pitch perception. Manuscript in preparation.

Deutsch, D., Moore, F. R., \& Dolson, M. (1984). Pitch classes differ with respect to height. Music Perception, 2, 265-271.

Deutsch, D., Moore, F. R., \& Dolson, M. (1986). The perceived height of octave-related complexes. Journal of the Acoustical Society of America, 80, 1346-1353.

Dowling, W. J., \& HaRwood, D. L. (1986). Music cognition. New York: Academic Press. 
ForTe, A. (1973). The structure of atonal music. New Haven: Yale University Press.

Geschwind, N., \& Fusillo, M. (1966). Color-naming defects in association with alexia. Archives of Neurology, 15, 137-146.

Humphreys, L. F. (1939). Generalization as a function of method of reinforcement. Joumal of Experimental Psychology, 25, 361-372.

KruMhansL, C. L. (1979). The psychological representation of musical pitch in a tonal context. Cognitive Psychology, 11, 346-374.

LoCkhead, G. R., BYRD, R. (1981). Practically perfect pitch. Journal of the Acoustical Society of America, 70, 387-389.

MEYer, M. (1904). On the attributes of the sensations. Psychological Review, 11, 83-103.

MEYeR, M. (1914). Review of G. Revesz, "Zur Grundleguncy der Tonpsychologie." Psychological Bulletin, 11, 349-352.

Moore, F. R. M. (1982). The computer audio research laboratory at UCSD. Computer Music Journal, 6, 18-29.

NeTtL, B. (1956). Music in primitive culture. Cambridge, MA: Harvard University Press.

Piston, W. (1941). Harmony. New York: Norton.

Pollack, I. (1978). Decoupling of auditory pitch and stimulus frequency: The Shepard demonstration revisited. Joumal of the Acoustical Society of America, 63, 202-206.

Revesz, G. (1913). Zur Grundleguncy der Tonpsychologie. Leipzig: Feit.

Risset, J. C. (1971). Paradoxes de hauteur: Le concept de hauteur sonore n'est pas le même pour tout le monde. Seventh Intemational Congress of Acoustics, Budapest, S10, p. 20.

Ruckmick, C. A. (1929). A new classification of tonal qualities. Psychological Review, 36, 172-180.
SCHRoEder, M. R. (1986). Auditory paradox based on fractal waveform. Journal of the Acoustical Society of America, 79, 186-188.

ShePARD, R. N. (1964). Circularity in judgements of relative pitch. Journal of the Acoustical Society of America, 36, 2345-2353.

SHEPARD, R. N. (1965). Approximation to uniform gradients of generalization by monotone transformations of scale. In D. I. Mostofsky (Ed.), Stimulus generalization. Stanford: Stanford University Press. SHEPARD, R. N. (1982). Structural representations of musical pitch. In D. Deutsch (Ed.), The psychology of music. New York: Academic Press.

Terhardt, E., \& SeEwann, M. (1983). Aural key identification and its relationship to absolute pitch. Music Perception, 1, 63-83.

TerhardT, E., \& WARD, W. D. (1982). Recognition of musical key: Exploratory study. Joumal of the Acoustical Society of America, 72, 26-33.

VON EHRENFELS, C. (1890). Über Gestaltqualitäten. Viertjahrshrift für Wissenschafiliche Philosophie, 14, 249-292.

WARD, W. D., BuRNS, E. M. (1982). Absolute pitch. In D. Deutsch (Ed.), The psychology of music. New York: Academic Press.

\section{NOTES}

1. As appropriate, envelopes will be referred to by their spectral peaks. Thus, for example, the term "C4 envelope" will be used to refer to the envelope with spectral peak at C4, and so on. In addition, in Experiment 1 , the term " $C$ envelope" will be used to refer to envelopes with spectral peaks either at $\mathbf{C 4}$ or at $\mathbf{C 5}$.

2. For all analyses, the alpha level was set at .01 . 